\title{
Heparin release from thermosensitive hydrogels
}

\author{
Anna Gutowska ${ }^{\mathrm{a}}$, You Han Bae ${ }^{\mathrm{a}}$, Jan Feijen ${ }^{\mathrm{b}}$ and Sung Wan Kim ${ }^{\mathrm{a}}$ \\ - Department of Pharmaceutics and Center for Controlled Chemical Delivery, University of Utah, Salt Lake City, Utah, USA: \\ 'Department of Chemical Technology, University of Twente. Enschede. Netherlands
}

(Received 21 February 1992; accepted in revised form 1 May 1992)

Thermosensitive hydrogels (TSH) were synthesized and investigated as heparin releasing polymers for the prevention of surface induced thrombosis. TSH were synthesized with $\mathrm{N}$-isopropyl acrylamide (NiPAAm) copolymerized with butyl methacrylate (BMA) (hydrophobic) or acrylic acid (AAc) (hydrophilic) comonomers. The incorporation of hydrophobic and hydrophilic comonomers strongly influences the swelling/shrinking behavior of TSH. Upon deswelling, gels containing the hydrophobic comonomer formed a skin-type layer, which acted as a rate controlling membrane, while the hydrophilic comonomer greatly increased gel swelling, relative to NiPAAm. Equilibrium swelling in isotonic PBS and deswelling kinetics of the synthesized gels were examined at various temperatures. The loading of heparin into the different gels was studied as a function of temperature, solution concentration, and gel composition. The release kinetics of heparin was found to be influenced by gel composition and loading temperature; the release correlated with the gel deswelling kinetics. In the case of $\mathrm{Ni}$ PAAm/BMA gel, the release protile of heparin was affected by temperature dependent properties of the skin-type diffusional barrier formed on the gel surtace.

Key words: Thermosensitive hydrogels; Heparin releasing polymers; Poly (N-isopropylacrylamide)

\section{Introduction}

Thermosensitve hydrogels (TSH) exhibit temperature dependent swelling in water due to the fact that their polymer-polymer and polymer-water interactions are strongly influenced by temperature. Hydrogels based on $\mathrm{N}$-isopropyl acrylamide (NiPAAm) demonstrate a negative thermosensitivity i,e., they are highly swollen at low temperatures and deswell significantly with increasing temperature [1]. An abrupt change in swelling is observed around $32^{\circ} \mathrm{C}$, the temperature corresponding to the lower critical solution

Correspondence to: \$.W. Kim, Dept. of Pharmaceutics and Center for Controlled Chemical Delivery, University of Utah, 421 Wakara Way 318, Salt Lake City, UT 84108, USA. temperature (LCST) of poly (NiPAAm) [2]. At this temperature the entropy driven release of water molecules, structured around the hydrophobic isopropyl side chains, leads to the gel deswelling.

Recently, thermally reversible polymers and hydrogels applied in therapeutics and diagnostics were reviewed by Hoffman [3]. Okano et al. [4] discussed the application of temperature responsive membranes for controlled drug delivery. Utilization of TSH gels in molecular separations $[5,6]$ enzyme activity controlling systems [7], and as a tissue culture substrates [8] have also been described. Okano et al. [9] and Bae et al. $[10,11]$ demonstrated an "on-off" release profile in response to a stepwise tempera- 
ture change with NiPAAm based TSH modified with hydrophobic components. The "on-off" Ielease profile was explained by the formation of a dense, less permeable surface layer of gel, described as a skin-type barrier. Formation of the skin was confirmed by the presence of water pockets on the disk surface, shown by optical microscopy. Surface skin layer formed upon a sudden temperature change, through gel shrinking temperature, because the gel surface deswelled and collapsed faster than the interior.

We propose the use of TSH as heparin releasing polymers for the prevention of surface induced thrombosis. Surface induced thrombosis occurs when a polymer surface contacts blood, stimulating the intrinsic clotting pathway [12]. One method to improve the properties of a blood contacting surface is the application of heparin releasing polymers [13,14]. Heparin, a well known anticoagulant, prevents fibrin formation by catalyzing the inactivation of thrombin by ATIII. Systemic administration of heparin, however, may cause serious side effects, such as thrombocytopenia and prolonged bleeding. Controlled release of heparin from the surface of a blood contacting device is advantageous over systemic administration because optimum anticoagulant activity is achieved at the blood-polymer interface with much smaller doses of drug, thereby decreasing side effects.

Heparin releasing polymers, studied so far, may be divided into two main types, ionically bound heparin polymers and polymers containing physically dispersed heparin. The first class of materials involves polymers with surface bound cationic groups [15-17]. Heparin is released from these polymers via an ion exchange mechanism with the ions circulating in blood. It has been shown, however, that positively charged surfaces exposed to blood may cause adverse reactions with platalets [18]. Therefore, to maintain the antithrombogenic properties of the polymeric surface, these materials require a relatively high heparin release rate, nearly $2.4 \mu \mathrm{g} /$ $\mathrm{h} / \mathrm{cm}^{2}$, as det . ...ined by Tanzawa et al. [17].

Materials containing heparin physically dispersed within the matrix exhibit antithrombo- genic properties with a lower heparin release rate from the polymer. Silicone rubber containing physically dispersed heparin was examined by Hufnagel [15]. Ebert et al. [20] synthesized poly (hydroxyethyl methacrylate) (p-HEMA) with dispersed heparin and prostaglandin. Their results showed that drug release from the gel matrix continued for $10 \mathrm{~h}$, and $25 \%$ of heparin remained entrapped within the network. Lin et al. [21] dispersed heparin within a polyurethane network, and demonstrated patency of a rabbit arterio-arterio shunt for $5 \mathrm{~h}$ with a minimum heparin release rate of $0.24 \mu \mathrm{g} / \mathrm{h} / \mathrm{cm}^{2}$.

The rationale of this manuscript is based on the properties of TSH offering unique flexibility as to the amount and kind $n$ f anticoagulant drug(s) which can be loaded into the gel. A TSH, exhibiting negative thermosensitivity, when immersed into a heparin loading solution at low temperature would swell and adsorb the drug. At higher (body) temperature, the swollen gel would collapse and release heparin (Fig. 1). An initial rapid release of heparin, due to the squeezing effect of the collapsing polymer, is expected to be followed by a slow release of drug controlled by solute diffusion within the collapsed network.

Our gels are design to prevent surface induced thrombosis for short term applications (few days of contact with blood). The initial events occuring on the blood contacting surface are very important in controlling surface induced thrombosis. These events include almost immediate adsorption of the protein layer (within $5 \mathrm{~min}$ ) and the subsequent activation of the intrinsic clotting pathway. If not prevented, thrombosis

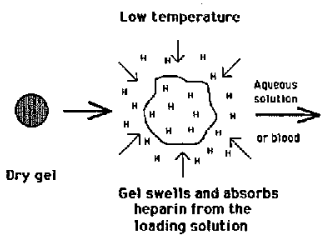

High temperoture

Fig. 1. Schematic representation of heparin loading and release from thermosensitive hydrogels. 
occurs within minutes upon the contact of blood with a polymer surface. Initial local high concentrations of heparin at the blood contacting surface could be essential for deactivation of clotting factors. The subsequent slow release rate of heparin is expected to prevent surface induced thrombosis for up to 6 days. Future in vivo experiments will determine how long the beparin releasing system is bioactive.

Heparin releasing TSH, based on NiPAAm copolymers with butyl methacrylate (BMA) or acrylic acid (AAc) were designed. BMA comonomer, a hydrophohic component, was incorporated to study the influence of the skin-type diffusional barrier on the heparin release kinetics. AAc comonomer, a hydrophilic component, was added to increase gel swelling levels at physiological temperature. The following properties of synthesized gels were investigated: equilibrium swelling, deswelling kinetics, loading and release characteristics. Loading and release studies were conducted using commercial polydisperse heparin. The influence of the loading temperature on heparin release kinetics from gels containing BMA and AAc comonomers was also described.

\section{Materials and Methods}

\section{Materials}

$\mathrm{N}$-isopropylacrylamide (NïPAm), obtained from Eastman Kodak Company, was recrystallized from hexane. Acrylic acid (AAc), obtained from Aldrich, was puriffed by distillation under reduced pressure; iractions collected at $42-$ $44^{\circ} \mathrm{C} / 13 \mathrm{~mm} \mathrm{Hg}$ were used. Butylmethacrylate (BMA) was obtained from Polysciences, Inc. (Warrington, PA) and purified by distillation under nitrogen, the fraction at $57^{\circ} \mathrm{C} / 17 \mathrm{mmHg}$ was collected. Ethyleneglycol dimethacrylate (EC _ MA) was obtained from Polysciences and used as received. $2,2 *$-Azobis-izobutyronitrile (AIBN) was obtained from Eastman Kodak Company, and recrystallized from methanol. Dioxane, HPLC grade, was obtained from Aldrich and used as received. Polydisperse heparin sodium salt (mol. wt. 5,000-20,000), from porcine intestinal mucosa, was obtained from Diosynth, INC., and used as received. Isotonic phosphate buffer saline (PBS), $\mathrm{pH}=7.4$, was used as a release medium and to preparc loading solutions.

\section{Gel synthesis}

Poly(NiPAAm) gels were synthesized with 5 mole\% BMA (NiPAAm/BMA) or 2 mole\% AAc (NiPAAm/AAc) comonomers in $50 \mathrm{w} \%$ dioxane solution. EGDMA ( 1 mole\%) was used as a crosslinking agent, and AIBN ( $7.4 \mathrm{mmol} / \mathrm{l}$ ) was used as a free radical initiator. Reaction mixtures were bubbled with dry nitrogen for $20 \mathrm{~min}$ and then injected between two Teflon plates separated by a $1 \mathrm{~mm}$ thick rubber gasket. Polymerization was carried out at $65^{\circ} \mathrm{C}$ for $24 \mathrm{~h}$. Gels, obtained in the form of films, were immersed in distilled water at room temperature and cut into disk shape samples. Disks were then Soxblet extracted in ethanol for $48 \mathrm{~h}$, and dried under vacuum before use. Obtained dried disks were $6 \mathrm{~mm}$ in diameter and $0.4 \mathrm{~mm}$ thick.

\section{Swelling measurements}

Equilibrium swelling properties of NiPAAm/ BMA and NiPAAm/AAc gels were studied over a temperature range of $10-40^{\circ} \mathrm{C}$ in isotonic PBS. Disks were equilibrated for 3 days at each temperature to a constant equilibrium weight. Equilibrium swelling ratio, $S_{(\mathrm{eq})}$ was calculated from the following equation:

$S_{\text {(ea) }}=\left(W_{\mathrm{s}}-W_{\mathrm{d}}\right) / W_{\mathrm{d}}$

where $W_{s}$ denoles the weight of a swollen poly. mer, and $W_{d}$ denotes the weight of a dry polymer.

For deswelling kinetic studies, gels were equilibrated in isotonic PBS at 1,15 , and $20^{\circ} \mathrm{C}$ to constant weight. Swollen disks were immersed directly in PBS preequitibrated at $37^{\circ} \mathrm{C}$. The disks were removed from the $37^{\circ} \mathrm{C}$ buffer at predetermined times, the weight recorded, and the swelling ratio at time $t, S_{v}$, was calculated based on: 
$S_{t}=\left(W_{l}-W_{\mathrm{d}}\right) / W_{\mathrm{d}}$

where $W_{f}$ denotes the weight of a swollen polymer at time $t$.

\section{Heparin loading}

Heparin was loaded into NiPAAm/BMA and NiPAAm/AAc gels using solution sorbtion method. Heparin solutions $(1.25-10.0$ wt\%) were prepared in isotonic PBS $(\mathrm{pH}=7.4)$. Heparin was loaded into the gels at 1,15 , and $20^{\circ} \mathrm{C}$ for 3 days. Before the immersion in the release medium, the disks were briefly rinsed with PBS (same temperature as the loading solution), and the surface blotted with a damp filter paper. Total loading was determined after extensive extraction in $1{ }^{\circ} \mathrm{C}$ buffer solution. Loading percent was calculated as:

Load\% $=\left\{W_{\text {drug }} /\left(W_{\text {polymer }}+W_{\text {drug }}\right)\right\} \times 100$

\section{Heparin release}

Heparin release from disk shaped samples was conducted at $37^{\circ} \mathrm{C}$ in PBS at pH=7.4. No drying procedure was applied to the loaded disks. Swollen disks were taken out of the loading solution, briefly rinsed with PBS (temperature of the loading solution), and immersed directly in the release medium. To maintain sink conditons, discs were transferted into $10 \mathrm{ml}$ fresh PBS, preequilibrated at $37^{\circ} \mathrm{C}$, at predetermined times.

The amount of released heparin was measured by the Azure II colorimetric assay [22]. In this assay the absorption of heparin-Azure II complex is measured at $500 \mathrm{~nm}$. Samples $(0.5 \mathrm{ml})$ of the release medium were added to $4.5 \mathrm{ml}$ of 0.01 $\mathrm{mg} / \mathrm{ml}$ Azure II solution in water and vigorously mixed on the Vortex mixer. Absorption of the solution was measured after $1 \mathrm{~min}$, with water used as a reference.

Release profiles are presented as a fractional release of drug, and only the points corresponding to the release rates higher than $0.24 \mathrm{tg} / \mathrm{cm}^{2}$ ) hr are reported. Calculations of the release rates per surface area of the disk were based on disks dimensions at $37^{\circ} \mathrm{C}$. After each release experi- ment at $37^{\circ} \mathrm{C}$ the amount of drug that remained entrapped in the collapsed network was determined by extensive extraction of the disks at $1^{\circ} \mathrm{C}$ in PBS.

\section{Results and Discussion}

\section{Equiltbrium swelling properties}

The results of equilibrium swelling of $\mathrm{Ni}$ PAAm, NiPAAm/BMA and NiPAAm/AAc gels between $10-40^{\circ} \mathrm{C}$ in isotonic PBS are presented in Fig. 2. Gel composed of crosslinked NiPAAm deswells abruptly around $32^{\circ} \mathrm{C}$, i.e. at the LCST of poly (NiPAAm), due to the entropy driven release of water molecules structured around the hydrophobic isopropyl side chains. This temperature, at which the gel collappse is observed, might be defined as a gel collapse temperature (GCT). Above the GCT only very small changes in the equilibrium swelling ratio occur with increasing temperature. It is possible to modify the GCT of a get by copolymerization with more hydrophobic (HPB) or more hydrophilic (HPL) comonomers. HPB comonomer decreases the GCT, whereas the HPL comonomer increases the GCT.

As seen in Fig. 2, copolymerization of NiPAAm with BMA resulted in a lower GCT, as compared to the pure NiPAAm gel, and copoly. merization with $A A C$, resulted in an increased

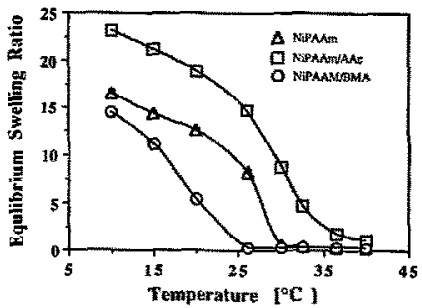

Fig. 2. Equilibrium swelling of NiPAAm, NiPAAm/BMA and NiPAAm/AAc in isotonic PBS. Error bars represent the standard deviation for $n=4$. For some data poinis error bars are smaller than the plot symbols. 
GCT. NiPAAm/AAc gel showed the highest equilibrium swelling at each temperature studied, due to the hydrophilic contribution of AAc. Based on the equilibrium swelling curve (Fig. 2), the NiPA Am $/ \Lambda \Lambda c$ gel at $37^{\circ} \mathrm{C}$ contained $52 \%$ water, while the NiPAAm and NiPPAm/BMA gels contained $20 \%$ and $18 \%$ water, respectively. This is a significant difference from the point of view of the diffusion of hydrophilic macromolecules, such as heparin, at physiological temperatures.

\section{Deswelling kinetics}

In the proposed application of İNiPAÁm based gels, heparin loading takes place at low temperatures (high swelling levels), and the release occurs at $37^{\circ} \mathrm{C}$ ( iow swelling levels). Upon the immersion of the loaded, swollen gel in the release medium at $37^{\circ} \mathrm{C}$, a significant volume change of the gel occurs due to deswelling. Hence, deswelling kinetics will greately influence release profilus.

Fig. 3 presents the deswelling kinetics for NiPAAm/BMA gel preequilibrated at three different temperatures, 1,15 , and $20^{\circ} \mathrm{C}$, and subsequently immersed at $37^{\circ} \mathrm{C}$. Disks swollen initially at $1^{\circ} \mathrm{C}$ collapsed to their equilibrium state

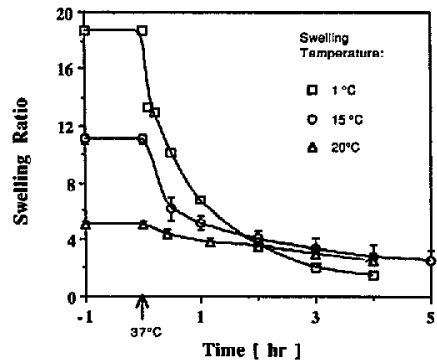

Fig. 3. Deswelling kinetics of NiPAAm/BMA gel equilibrated at three different temperatures $\left(1,15\right.$ and $\left.20^{\circ} \mathrm{C}\right)$ and immersed at $37^{\circ} \mathrm{C}$ at time " 0 ". Error bars represent the standard deviation for $\boldsymbol{n}=4$. For some data points error bars are smaller than the plot symbols. within $3 \mathrm{~h}$, whereas disks swollen initially at 15 and $20^{\circ} \mathrm{C}$ remain swollen above their equilibrium water content for $24 \mathrm{~h}$ and 7 days, respectively. These data suggest that, in the case of NiPAAm/BMA gel, the rate of the deswelling process depends on the temperature of the initial equilibration.

As described by Okano et al. [9] and E.S Matsuo [23], the sudden temperature change from 20 to $30^{\circ} \mathrm{C}$ leads to the formation of a dense, less permeable surface layer of NiPAAm/BMA gel, described as a skin-type barrier. Formation of the skin was confirmed by the presence of water pockets on the disk surface, shown by optical microscopy. A possible explanation for the surface skin layer formation is that upon a sudden temperature change, through GCT, the gel surface collapses faster than the bulk matrix.

In the present study, swollen NiPAAm/BMA gel was exposed to even larger temperature changes, from 1 to $37^{\circ} \mathrm{C}$ and from 15 to $37^{\circ} \mathrm{C}$ (Fig. 3). These results suggest that the properties of the skin-type barrier are influenced by the temperature difference between the initial and final medium. This temperature difference, in turn, determines the difference between the initial and final equilibrium swelling degrecs $\left(\Delta \mathrm{S}_{e q}\right)$. In cases where $\Delta \mathbf{S}_{\text {eq }}$ is large, a significant hydrostatic pressure will build within the swollen disk. The high hydrostatic pressure of the entrapped water expands the water pockets, and only a thin and permeable skin will form on the surface. This temperature dependenci of skin permeability lead to an observation that the release rate from swollen NiPAAm/BMA gels can be controlled by optimmizing the initial loading temperature.

Fig, 4 shows the results of the deswelling kinetic studies of NiPAAm/AAC gel. Here, the deswelling profiles are independent of the initial temperature. NiPAAm/AAc gel showed the same rate of deswelling at $37^{\circ} \mathrm{C}$, after equilibration at 1,15 or $20^{\circ} \mathrm{C}$. It can be concluded that free water efflux occurred in this case, and that a skin-type barrier did not form on the surface of a gel containing hydrophilic comonomer. 


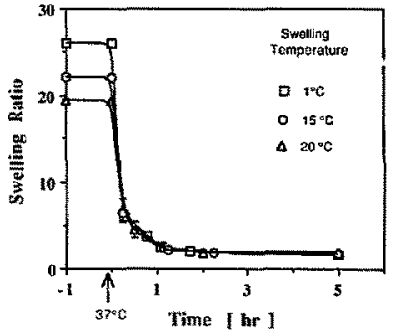

Fig. 4. îeswelling kinetics of NiPAAm/AAc gel equilibrated at three different temperatures $\left(1,15\right.$ and $\left.20^{\circ} \mathrm{C}\right)$ and immersed at $37^{\circ} \mathrm{C}$ at time " 0 ". Eiror bars represent the sandard deviation for $n=4$. For some data points error bars are smatter than the plot symt ols.

\section{Loading studies}

The following factors controling heparin loading into NiPAAm based gels were examined: loading solution concentration, loading temperature, and the effect of comonomers. Fig. 5 illustrates the effect of the loading solution concentration on the loading pcreent of polydisperse heparin. NiPAAm/AAc gel was loaded with heparin at $15^{\circ} \mathrm{C}$ in isotonic PBS solutions containing 1.25-10 wt\% heparin. A nonlinear loading profile at increasing concentration was observed, with the highest loading percent corresponding to 4-6 wt\% heparin. Nonlinear loading can be explained by the corresponding swelling curve of NiPAAm/AAc gel in heparin loading solutions (Fig. 6), which shows that swelling of the gel decreases with increasing heparin concentration. Decreased swelling of gel with increasing solute concentration is due to the osmotic deswelling caused by decreased water activity in the solution. Hence, as the concentration of heparin increases, the gel is iess swollen, and the free volume available for the diffusion of macromolecules is smaller. Heparin molecules with the highest molecular weight are probably excluded from gel and are the reason for an additional osmotic deswelling. A similar phenomenon was observed by Bastide et al. [24], who

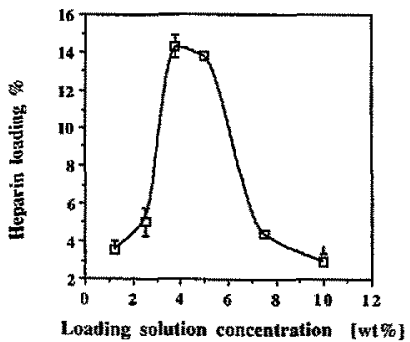

Fig. 5. Heparin loading into NiPAAm/AAC gel at $15^{\circ} \mathrm{C}$. The effect of loading suiwtion concentration on the loading per cent of drug. Error bars represent the standard deviation for $n=3$.

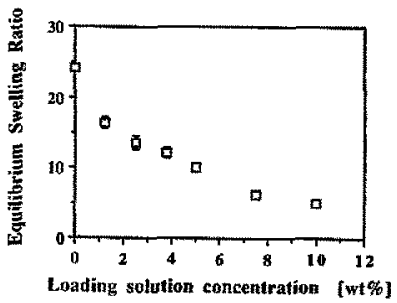

Fig. 6. Equilibrium swelling of NAPAAm/AAc gel in loadint: solutions of heparin. Error bars represent the standard deviation for $n=3$. For some data points error bars are smaller than the plot symbols.

described an osmotic deswelling of polymeric gels in solutions of macromolecules.

The eflect of comonomers (AAc vs. BMA) and the loading temperature on heparin content in NiPAAm based gels is illustrated in Fig. 7. As shown, NiPAAm/AAc gel gave higher heparin loading percents at each temperature, compared to NiPAAm/BMA. Heparin content decreased significantly with increasing temperature for both gels. These results suggest that heparin loading depends on the gel equilibrium swelling. Since heparin and the NiPAAm/AAc gel are negaively charged, the loading is expected to be af- 


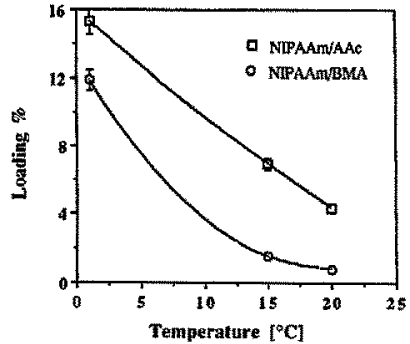

Fig. 7. Heparin loading into NiPAAm/BMA and NiPAAm/ AAcgels. Loading temperature and comonomer effect. ( 5 wt\% heparin loading solution in PBS was used).

fected by the ionic (repulsive) solute-matrix interactions. However, these experiments were conducted in isotonic PBS, and the concentration of ions is sufficient to screen the effect of charges. Therefore, based on the results presented in Fig. 7 it was assumed that in both NiPAAm/AAc and NiPAAm/BMA gels, heparin loading was controlled by the equilibrium swelling of gels.

In order to check whether heparin was actually diffusing into the core of the disks, loading experiments were performed with disks with different thicknesses. The results showed that comparable londing percentages $( \pm 2 \%$ ) were obtained with disks having the same radius, but differing by a factor of two in thickness. Therefore, it can be concluded that disks are loaded homogeneously.

\section{Release studies}

Heparin release from NiPAAm thermosensitive hydrogels was studied as a function of loading temperature $\left(1,15\right.$, and $\left.20^{\circ} \mathrm{C}\right)$ and a comonomer effect (BMA vs AAc). The release profiles presented in Figs. 8 and 9, show an initial high heparin release rate, followed by a significantly slower rate at fater times. This suggests that initially, due to the dynamic squeezing of the collapsing polymer network, drug release was a result of a convective flow of heparin and water.
Later, at low swelling levels of gel, release was controlled by the diffusion of heparin from the collapsed network.

The effect of loading temperature was studied to verify the hypothesis suggested by the deswelling kinetics studies. That is, the release rate can be controlled by the iemperature dependent skintype barrier which forms on the surface of the NiPAAm/BMA gel. Fig. 8 illustrates the effect of the lnading temperature on the heparin release rate from the NiPAAm/BMA gel. Disks loaded with heparin at 1,15 or $20^{\circ} \mathrm{C}$ were immersed in the $37^{\circ} \mathrm{C}$ release medium. Obtained release profiles are the following: disks loaded at $1^{\circ} \mathrm{C}$ released $98 \%$ of heparin within $8 \mathrm{~h}$, disks loaded at $15^{\circ} \mathrm{C}$ showed a more gradual release profile and released $95 \%$ of drug within $18 \mathrm{~h}$, disks loaded at $20^{\circ} \mathrm{C}$ showed no heparin release at $37^{\circ} \mathrm{C}$. In each case, all of the drug, which remained entrapped in the NiPAAm/BMA network at $37^{\circ} \mathrm{C}$ was released at $1^{\circ} \mathrm{C}$. The various heparin release profles obtained after loading at different temperatures can be explained by considering the equilibrium swelling ratio of a gel in the loading solution and the percent loading of drug, as sum. marized in Table 1. A comparison of these valwes with the release profiles presented in Fig. 8 , leads to the conclusion that a higher initial equilibrium swelling combined with a higher loading

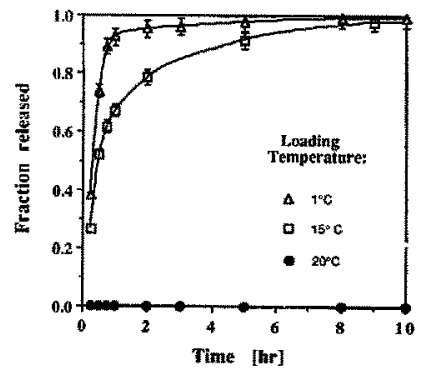

Fig. 8. Heparin release from NiPAAm/BMA gal at $37^{\circ} \mathrm{C}$ after loading at three different temperatures $\left(1,15\right.$ and $\left.20^{\circ} \mathrm{C}\right)$. Effect of the londing temperature on the heparin release kinetics. (Error bars represent the standard deviation for $n=3$ ). 
TABLE 1

Loading of heparin into NiPAAm/BMA gel. Heparin loading percentages and the comesponding equilibrim swelling ratios of gel in 5 wo heparin loading solution at 1,15 and $20^{\circ} \mathrm{C}$.

\begin{tabular}{lll}
\hline Temperature & $\begin{array}{l}\text { Heparin } \\
\text { loading }(\%)\end{array}$ & $\begin{array}{l}\text { Swelling matio } \\
\text { in heparin } \\
\text { loading solution }\end{array}$ \\
\hline $1{ }^{\circ} \mathrm{C}$ & $11.9 \pm 0.2$ & $9.7 \pm 0.4$ \\
$15^{\circ} \mathrm{C}$ & $1.5 \pm 0.3$ & $5.3 \pm 0.1$ \\
$20^{\circ} \mathrm{C}$ & $0.6 \pm 0.2$ & $1.8 \pm 0.1$ \\
\hline
\end{tabular}

percent leads to the faster release rate. This conclusion correlates well with the results of the deswelling kinetics studies, where the higher initial equilibrium swelling resulted in a faster deswelling of NiPAAm/BMA gel (Fig. 3).

In the case of heparin release, however, the effect of the hydrophilic drug on the properties of the skin-type barrier should be also considered. Heparin attracts water and provides a more hydrophilic enviromment within the disk. It is thus possible that the properties of the rate controlling barrier are influenced by both the temperature gradient between the loading and release medium and by the content of a hydrophilic drug within the disk. Therefore, after loading at $1^{\circ} \mathrm{C}$, (the highest ecuilibrium swelling and the highest heparin losd percent ) the thinnest and the most permeable skin is formed on the disk surface at $37^{\circ} \mathrm{C}$, ind as a consequence the fastest release is observed. Loading at $15^{\circ} \mathrm{C}$ results in a lower equilibrium swelling of the gel and also lower heparin content than those at $1^{\circ} \mathrm{C}$. Hence, after loading at $15^{\circ} \mathrm{C}$, a less permeable skin formed on the disk surface at $37^{\circ} \mathrm{C}$, and a more gradual release profile of heparin was observed. These results show that in the case of NiPAAm/ BMA gel, loading temperature can be used as a variable tc control the heparin release rate.

After loading at $20^{\circ} \mathrm{C}$, NiPAAm/BMA gel showed no rclease of heparin at $37^{\circ} \mathrm{C}$, suggesting the presence of a dense skin on the disk surface. These results are consistent with the results obtained by Hae et al. [10], who showed that by changing the temperature of the release medium from 20 to $30^{\circ} \mathrm{C}$ the release of drug stoped, due to the formation of a skin-type barrier on the surface.

Heparin release profiles from NiPAAm/AAc gel loaded at 1,15 and $20^{\circ} \mathrm{C}$ are presented in the Fig. 9. Loading percentages and the corresponding equilibrium swelling ratios in loading solutions are summarized in Table 2. All release profiles show similar kinetics, an initial rapid release due to the gel squeezing effect and a subsequent slow release of heparin controlled by the rate of solute diffusion through the collapsed network. Slightly higher release rates were obtained from disks loaded at 1 and $15^{\circ} \mathrm{C}$ due to the higher loading percentages of heparin at these temperatures. It could also be noted that the initial rapid

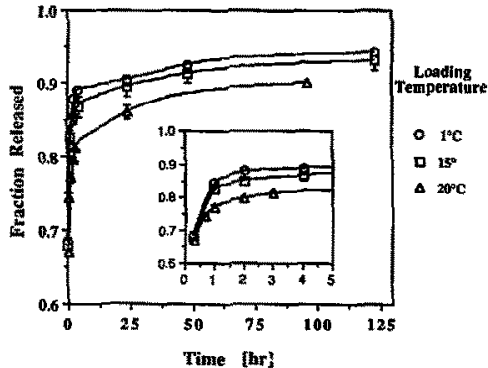

Fig. 9. Heparin release from NiPAAm/AAc gel at $37^{\circ} \mathrm{C}$ after loading at three different temperatures $\left(1,15\right.$ and $\left.20^{\circ} \mathrm{C}\right) \mathrm{Ef}$ fect of the loading temperature on the hepariu release kinet. ics. The small inserted figure shows early times of the release. 0 to $5 \mathrm{~h}$. (Error bars represent the standard devietion for $n=3$ ).

TABLE 2

Lotding of heparin into NiPAAm/AAc gel. Heparin loading percentages and the corresponding equilibrium swelling $\mathrm{ra}$ tios of gel in 5 wt\% heparin loading solution at 1,15 and $20^{\circ} \mathrm{C}$

\begin{tabular}{ccc}
\hline Temperature & $\begin{array}{l}\text { Heparin } \\
\text { loading } \\
\text { (0. }\end{array}$ & $\begin{array}{l}\text { Swelling ratio } \\
\text { in heparin } \\
\text { loading solution }\end{array}$ \\
\hline $1{ }^{\circ} \mathrm{C}$ & $15.3 \pm 0.2$ & $13.2 \pm 0.1$ \\
$15^{\circ} \mathrm{C}$ & $6.9 \pm 0.3$ & $9.3 \pm 0.1$ \\
$20^{\circ} \mathrm{C}$ & $4.3 \pm 0.2$ & $7.9 \pm 0.1$ \\
\hline
\end{tabular}


release, which lasted for about $1 \mathrm{~h}$, corresponded to the initial rapid deswelling of NiPAAm/AAc gel (Fig. 4). As was discussed, deswelling profiles of the NiPAAm/AAc gel equilibrated at 1, 15 and $20^{\circ} \mathrm{C}$ overlaped and did not show temperature dependence. Correlation between the deswelling curves and the heparin release profiles is in agreement with our hypothesis that the NiPAAm/AAc gel does not form a skin-type barrier on the surface, due to the presence of the hydrophilic comonomer.

A direct evaluation of the comonomer effect on the heparin release kinetics is illustrated by comparing the heparin release profiles of $\mathrm{Ni}$ PAAm/BMA and NiPAAm/AAc gels loaded at the same temperature. Heparin release profile from NiPAAm/BMA gel loaded at $15^{\circ} \mathrm{C}$ is presented in Fig. 8, and the corresponding profile for NiPAAm/AAc gel in Fig. 9. After the initial squeezing, NiPAAm/BMA gel continued to release heparin for $15 \mathrm{~h}$, whereas the NiPAAm! AAC gel showed a prolonged slow release of heparin for $124 \mathrm{~h}$. This difference is related to the higher GCT of NiPAAm/AAc gel and higher swelling levels at $37^{\circ} \mathrm{C}$. Equilibrium water content of NiPAAm/AAc gel at $37^{\circ} \mathrm{C}(52 \%)$ allows for diffusion of heparin macromolecules which remained in the gel network after the initial squeezing. NiPAAm/BMA gel contained only $20 \%$ water after $15 \mathrm{~h}$ at $37^{\circ} \mathrm{C}$ and also a dense skin was present on the disk surface, therefore, no further heparin release was observed.

The shape of the obtained release profiles is consistent with the behavior of swollen thermosensitive gels above their GCT. Upon a sudden temperature change, the collapsing polymer network squeezes the drug, and an initial rapid release is observed. This initial rapid release is followed by a slow release, where the ding release rate is controlled by the diffusion of solute within the collapsed network (NiPAAm/AAc gel), and/ or by the presence of the skin-type rate controlling barrier (NiPAAm/BMA gel).

\section{Conclusions}

NiPAAm based thermosensitive hydrogels containing HPB or HPL comonomers were ob- tained, and their appication as heparin releasing polymers were examined.

Equilibrium swelling studies showed that incorporation of BMA (HPB comonomer) resulted in a lowering of the GCT of NiPAAm gels, and incorporation of AAc (HPL comonomer) resulted in the increase of the GCT.

Deswelling kinetics studies revealed that in the case of NiPAAm/BMA gel, the rate of deswelling depended on the temperature of the initial equilibration. This was due to the formation of a skin-type rate controlling barrier on the gel surface. NiPAAm/AAc gel showed the same rate of deswelling, independent of the temperature of the initial equilibration. It was coneluded that a skintype barrier does not form on the surface of NiPAAm gel containing a hydrophilic comonomer.

Loading studies showed that the amount of heparin loaded into NiPAAm/BMA and NiPAAm/AAc was controlled by the equilibrium swelling ratio of geis in the drug solution. Nonlinear loading with increasing solution concentration was observed, with maximum loading corresponding to a 4-6 wt\% heparin solution.

Studies of the heparin release from swollen NiPAAm/BMA and NiPAAm/AAc gels revealed that release profiles correlated with deswelling kinetics of gels. The release profiles showed two distinct kinetics controlled by different mechanisms: an initial rapid release due to the network collapse (squeezing effect) and the subsequent slow release controlled by the rate of solute diffusion within the collapsed matrix, and /or by the presence of a rate controlling barrier

NiPAAm/AAc gels were obtained which released heparin with a rate greater than $0.24 \mu \mathrm{g} /$ $\mathrm{cm}^{2} / \mathrm{hr}$ for $124 \mathrm{~h}$. In the case of NiPAAm/BMA gel, the release rate was controlled by the temperature dependent properties of the skin-type rate controlling barrier which formed on the gel surface. This allowed for control of the release rate by changing loading temperature only, without polymer modification.

The results demonstrate loading and release of heparin using NiPAAm based TSH. These gels 
may be applied as a polymeric coatings on the surface of a blood contacting devices for prevention of the surface induced thrombosis.

\section{Acknowledgements}

The authors wish to thank Dr. H. Jacobs for hclpful discussions. This work was supported by NIH grant HL 17623-17.

\section{References}

1 L.D. Tayior and L.,D. Cerankowski, Preparation of films exhibiting a balanced temperature dependence to permeation by aqueaus solutions: a study of lower consolute behavior, J. Polym. Sci. Polym. Chem. 13 (1975) $2551-2570$.

$2 M$. Heskins and J.E. Guillet, Solution properties of poly (N-isopropyl acrylamide), J. Macromal. Sci.-Chem. A2 8 (1968) 1441-1455.

3 A.S. Hoffman, Application of thermally reversible polymers and hydrogels in therapeutics and diagnostics, 3 . Controlled Release 6 (1987) 297-305.

4 T. Okano, Y.H. Bae and S.W. Kim, Temperature responsive controlled orug delivery, in: J. Kast (Ed.), Pulsed and Self-Regulated Drug Delivery, CRC Press, Boca Raton, 1990, pp. 17-46.

5 R.F.S. Freitas and E.L. Cussler, Temperature sensitive gels as eatraction solvents, Chem. Eng. Sci, 42 (1987) 93...103.

6 H. Feil, Y.H. Bac, J. Fejjen and S.W. Kim, Molecular separation by thermosensitive hydrogel membranes, $J$. Mernbr. Sci. 64 (1991) 283-294,

7 T.G. Park and A.S, Hofman, tmmobilization of b-galactosidase in thermally reversible hydrogel beads, $J$. Biomed. Mater. Res. 24 (1990) 21-38.

8 T. Takezawa, Y* Mori and K. Yoshiza10, Cell culture on a thermoresponsive polymer surface, Biotechnology 8 (1990) 854-857.

9 T. Okano, Y.H. Bae, H. Jacobs and S.W. Kim, Thermally on-of switching polymers for drug perneation and release, J. Controlied Release 11 (1990) 255-265.

10 Y.H. Bae, T. Okano and S.W. Kim, "On-of?" control of solute transport. 1.Temperature dependence of swelling of $\mathrm{N}$-isopropyhacrylamide networks modiffed with hydrophobic components in water, Pharm. Res. \& (1991) $\$ 31-537$.
11 Y.H. Bae, T. Okano and S.W. Kim, "On-off" control of solute transport. Il. Solute release from thermosensitive hydrogels, Pharmaceutical Research 8 (1991) 624-628.

$12 \mathrm{M}$. Szycher, Thombosis, hemostasis and thrombolysis at prosthetic interfaces, in: M. Szycher (Ed.), Biocom* patible Polymers, Metals and Composites, Technomic Publishing Co., 1983, pp. i-35.

13 C.D. Ebert and S.W. Kim, Heparin/polymers for the prevention of surphaee thrombosis, in: R.S. Langer and D.L. Wise (Eds.), Medical Applications of Cortrolted Release, CRC Press, Boca Raton, 1984, vol. 2 pp. 77106.

14 J.C. McRea and S.W. Kim, Controlled release of bioactive agents for the designe of blood compatible polymers, in: M. Szycher (Ed.), Biocompatible Polymers. Plenum Press, 1983, pp.597-620.

Is V.L. Gott. J.D. Whiffen and R.C. Datton, Heparin bonding on colloidal graphite surfaces, Science 142 (1963) 1297-30.

16 R.I. Leininger and C.W. Cooper, Nonthrombogenic plastic surfaces, Science 1\$2 (1968) 1625-27.

17 H. Tanzawa, Y. Mori and N. Harumiya, Preparation and evaluation of a new athrombogenic heparinized hydrophilic polymer for use in cardio-vascular systems, Trans. Am. Soc. Arif. Intern. Organs 14 (1973) 188-194.

18 R. Shibuta, M. Tanaka, M. Sisido and Y. Imanishi, Synthesis of novel polyaminoetherurethaneureas and development of antithrombogenic material by their chemical modifications, J. Biomed. Mater. Res. 20 (1986) $971-987$.

19 C.A. Hufnagel, P.W. Conrad and J.F. Gillespie, Characteristics of materials for intravascular applications, Ann. N,Y. Acad. Sci. 146 (1968) 262-266.

20 C.D. Ebert, 3.C. McRea and S.W, Kim, Controlled release of anthithrombotic agents from polymer matrices, in: J. Baker (Ed.), Controlled Retease of Bioactive Materials, Academic Press, New York, 1980, pp. 107-119.

21 J.Y. Lin, C. Nojiri, T. Okano and S.W. Kim, Minimum heparin release rate for nonthrombogenicity, A.S.A.1.O. $101987602-605$.

22 S. Lam and R. Silben, Biochim. Biophys. Res. Commun. 69 (1976) 570-576.

23 E.S. Matsuo and T. Tanaka, Kinetics of discontinuous volume phase transition of geis, J. Chem. Phys. 88 (1988) 427-431.

24 J, Bastide, S. Candau and L. Leibler, Osnot ic deswelling of gels by polymer sotuivis, Macromolecules 14 (1981) 719-726. 